Original Article

\title{
IN VITRO CALLUS INDUCTION AND COMPARATIVE GC-MS ANALYSIS OF METHANOLIC EXTRACTS OF CALLUS AND LEAF SAMPLES OF AMPELOCISSUS LATIFOLIA (ROXB.) PLANCH
}

\author{
DEEP CHHAVI ANAND, RISHIKESH MEENA, VIDYA PATNI
}

Tissue Culture and Biotechnology Laboratory, Department of Botany, University of Rajasthan, Jaipur 302004

Email: chhavianand03@gmail.com

Received: 10 Jun 2018 Revised and Accepted: 30 Jul 2018

\begin{abstract}
Objective: The aim of the present study was to develop a callus induction protocol and comparative study of therapeutic phytochemicals present in in vivo leaf and in vitro callus extracts through Gas Chromatography-Mass Spectrometry analysis.

Methods: Murashige and Skoog media was used as culture media for callus induction. In vitro callus induction protocol was developed by studying the effects of various plant growth regulators like auxin, 2, 4-D (2,4-dichlorophenoxyacetic acid), NAA (naphthalic acetic acid), alone and in combination with cytokinin BAP (benzyl aminopurine), on leaf and stem explants. The GC-MS analysis of Ampelocissus latifolia was carried out on Shimadzu QP-2010 plus with thermal desorption system TD 20 to study the phytochemical profile.

Results: In vitro callus induction protocol was developed for the plant and callusing was done from leaf and stem explants of Ampelocissus latifolia. The best result for callus induction was obtained using leaf explant, and callus production were maximum in Murashige and Skoog medium fortified with BAP $(0.5 \mathrm{mg} / \mathrm{l})$ and NAA (1.0 mg/l). Major compounds identified in the GC-MS analysis were Campesterol, Stigmasterol, Beta-Sitosterol, Docosanol, Dodecanoic acid, etc., in in vitro extract and Beta Sitosterol, Tocopherol, Squalene, Bergamot oil, Margarinic acid, Hexadecanoic acid, etc., in in vivo extract. The different active phytochemicals identified have been found to possess a wide range of biological activities, thus this analysis forms a basis for the biological characterization and importance of the compounds identified for human benefits.

Conclusion: This is the first report on callus induction in Ampelocissus latifolia. From the results obtained through the in vitro callus induction and its comparative GCMS analysis with in vivo extract, it is revealed that Ampelocissus latifolia contains various bioactive compounds that are of importance for phytopharmaceutical uses. The GCMS analysis revealed that the amount of Beta-sitosterol and 5-Hydroxymethylfurfural (HMF) was very high in in vitro extract as compared to in vivo extract.
\end{abstract}

Keywords: Ampelocissus latifolia, GC-MS, Growth regulators, Stigmasterol, Callus, Phytochemicals

(C) 2018 The Authors. Published by Innovare Academic Sciences Pvt Ltd. This is an open access article under the CC BY license (http://creativecommons.org/licenses/by/4.0/) DOI: http://dx.doi.org/10.22159/ijpps.2018v10i9.27879

\section{INTRODUCTION}

From ancient times, medicinal plants have been used extensively for their tremendous healing properties and health benefits. India has a treasure of medicinal plants due to the rich diversity in its agroclimatic conditions [1]. The curative properties of medicinal plants are mainly due to the presence of various secondary metabolites such as alkaloids, flavonoids, glycosides, phenols, etc. present in them [2]. Plant tissue culture plays an important role in the production of in vitro plants and in the manipulation of plants for improved performance. Media composition mainly the hormonal balance is an important factor influencing in vitro culture initiation and plant regeneration from explants [3].

The plant Ampelocissus latifolia (Roxb.) Planch. belonging to the family Vitaceae, is an example of ethnomedicinal plant species which is the source of natural dye and a range of traditional medicines that cure various diseases[4]. It is a large herbaceous climber, with a tuberous rootstock. The roots have been used for the treatment of snake bite and for its astringent effect. The decoction of the root is also used in chronic dysentry. The tribes of Bihar used this plant for muscular pains, sores and fractured bones [5]. The common name of the plant is "Jungli angoor" and other common name is "Panibel" in India. Juice of tender leaves is used in dental problems and as a detergent for indolent ulcers. The stem ash is applied abdominally for easy delivery in pregnancy $[6,7]$. Due to enormous medicinal properties, the plant has been used extensively. Therefore in vitro callus from leaves and stem have been raised using various growth regulators like 2, 4-D, NAA alone and in combination with BAP. In recent years, Gas Chromatography-Mass Spectrometry (GC-MS) has been applied unambiguously to identify the structures of different phytoconstituents in plant extracts and biological samples with great success, so further the methanolic leaf and callus extracts were subjected to evaluation for Gas Chromatography-Mass Spectrophotometry analysis to study the phytochemical profile. The unknown organic compounds in a complex mixture can be determined by interpretation and also by matching the spectra with reference spectra $[8,9]$. Thus, the phytochemical screening may be useful for the detection and isolation of various bioactive compounds which subsequently may lead to drug discovery and development from these plants in vitro.

\section{MATERIALS AND METHODS}

\section{Chemicals and reagents}

Mercuric chloride, bavistin, tween20 detergent were obtained from Sigma-Aldrich. Growth regulators like NAA, BAP, 2,4-D, and methanol were purchased from HiMedia. All chemicals were of analytical grade and used without any further purification. The GCMS analysis of Ampelocissus latifolia was carried out on Shimadzu QP-2010 plus with thermal desorption system TD 20.

\section{Collection and preparation of a plant extract}

The plant was collected from Baghdhara Nature Park, Udaipur, Rajasthan. Leaves and stem were collected and used as explants for callus induction.

For in vitro callus induction, fresh leaves and stem were collected from the plant and washed under running tap water for $10 \mathrm{~min}$. Then washed with mild detergent tween 20 with gentle and continuous shaking and surface sterilized using bavistin fungicide $0.1 \%(\mathrm{w} / \mathrm{v})$ for 60 seconds. The leaves were then washed thrice using distilled water. Under Laminar air hood, the explant was surface sterilized using $0.1 \%$ $(\mathrm{w} / \mathrm{v})$ mercuric chloride solution for 1 minute and then washed thrice with sterile distilled water ( 1 minute each). 
For GC-MS profiling, explants were washed thoroughly with water to remove dirt. They were dried in the shade and ground into powder with the help of a grinder. The dried material (5 grams) was soxhlet extracted using $80 \%$ methanol as a solvent for $24 \mathrm{~h}$. Both the extracts were air dried, and finally, weight was taken.

\section{Culture media and tissue culture}

Murashige and Skoog media was used as culture media for callus induction. MS media consists of various macronutrients, macronutrients, vitamins and organics required for in vitro growth of the plant [10] and fortified with $3 \%(\mathrm{w} / \mathrm{v})$ sucrose as a carbon source and solidified using agar $0.8 \%(\mathrm{w} / \mathrm{v})$. Various growth regulators like 2, 4-D, NAA alone and in combination with BAP were added, and then the $\mathrm{pH}$ of the media was adjusted to $5.8 \pm 0.1$.

The media was then autoclaved at $121{ }^{\circ} \mathrm{C}$ for $15 \mathrm{~min}$ at $15 \mathrm{psi}$ pressure. The surface sterilized leaves were cut from edges and then into small explants of size approximately $5 \mathrm{~mm}^{2}$ and cultured on MS media supplemented with different plant growth regulators. Cultured flasks were incubated in a culture chamber under suitable in vitro conditions. The temperature of the chamber was maintained at $25 \pm 2{ }^{\circ} \mathrm{C}$ using the air conditioner, and light intensity (1200 lux) was provided from fluorescent tubes (40 watt) and incandescent bulbs ( 40 watts). A photoperiod of $16 \mathrm{~h}$ light was provided inside the culture chamber.

\section{RESULTS AND DISCUSSION}

\section{Callus induction}

In vitro callus induction protocol was developed for the plant and callusing was done from leaf and stem explants of Ampelocissus latifolia. The leaves and stem were cut into small segments and used as explants. The effects of the different concentrations of growth regulators on callus formation were determined. These explants were cultured on callus induction medium i.e. Murashige and Skoog medium consisting of different concentrations of different auxins and cytokinin B. A. P. The different concentrations of 2, 4-D $(0.5,1.0,1.5,2.0 \mathrm{mg} / \mathrm{l})$, NAA $(0.5,1.0,1.5,2.0 \mathrm{mg} / \mathrm{l})$ and in combination $2,4-\mathrm{D}+\mathrm{BAP}(0.5+1.0$, $1.0+1.0,1.0+0.5,1.5+1.0 \mathrm{mg} / \mathrm{l})$ and NAA+BAP $(0.5+0.5,0.5+1.0$, $1.0+0.5,1.0+1.0 \mathrm{mg} / \mathrm{l})$ were used. The best result was obtained using leaf explant and callus production was maximum in Murashige and Skoog medium fortified with BAP (0.5 mg/l) and NAA $(1.0 \mathrm{mg} / \mathrm{l})$.
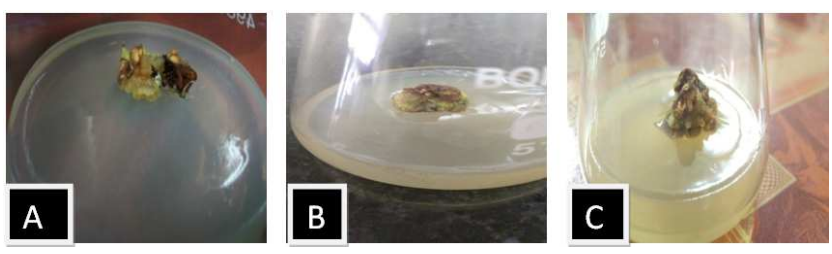

Fig. 1: Callus generated from leaf explant using different growth regulators, A-Callus induction from leaf supplemented with 2, 4-D (0.5 $\mathrm{mg} / \mathrm{l})$, B-Callus induction from leaf supplemented with NAA (1.0 mg/l), C-Callus induction from leaf supplemented NAA (1.0 mg/l) and BAP $(0.5 \mathrm{mg} / \mathrm{l})$
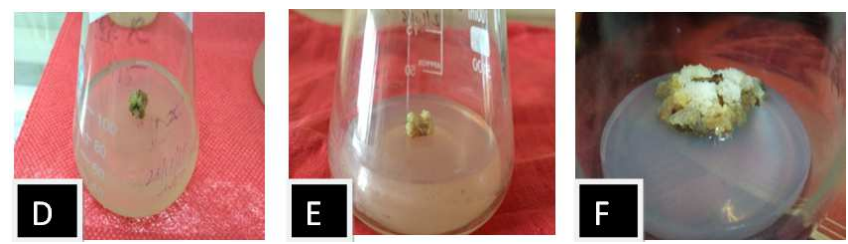

Fig. 2: Callus generated from stem explant using different growth regulators, D-Callus induction from stem supplemented with 2, 4-D (1.0 $\mathrm{mg} / \mathrm{l})$, E-Callus induction from stem supplemented with NAA (0.5 mg/l), F-Callus induction from stem supplemented NAA (1.0 mg/l) and BAP $(0.5 \mathrm{mg} / \mathrm{l})$

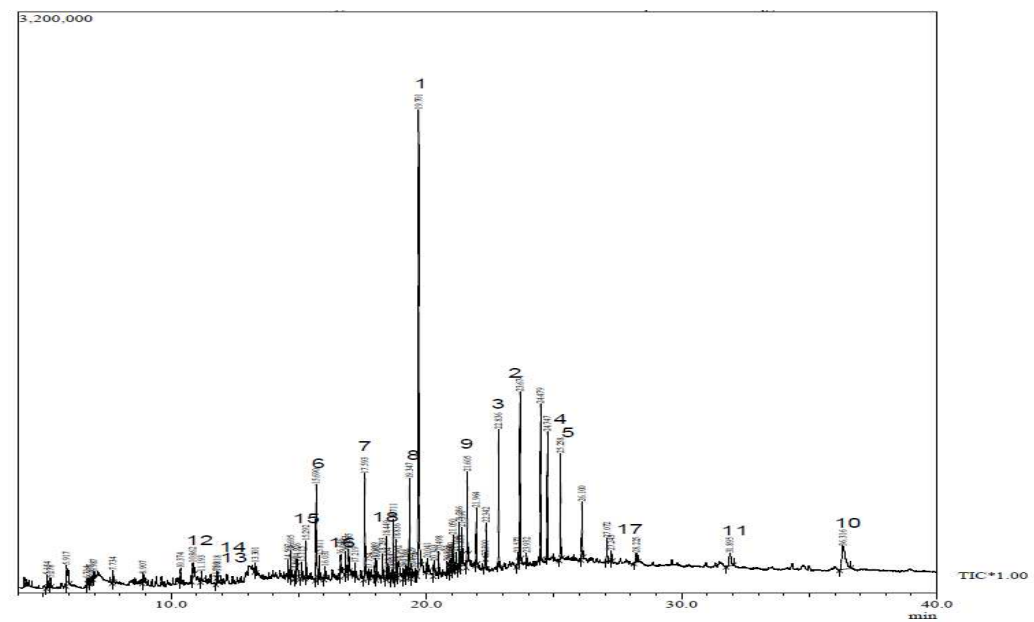

Fig. 3: GCMS chromatogram of methanolic leaf extract of Ampelocissus latifolia

\section{Phytochemical analysis by GC-MS}

GCMS analysis was done to determine the bioactive compounds from methanolic leaf and callus extracts of Ampelocissus latifolia. The analysis of GC-MS chromatogram (fig. 3 and 4) showed peaks of various useful phytochemical constituents present in methanolic extracts of Ampelocissus latifolia in vitro and in vivo. On comparison of mass spectra of phytoconstituents with the NIST library, various bioactive compounds were characterized and identified (table 1 and 2). 
Table 1: Major peaks identified in in vivo methanolic extract of Ampelocissus latifolia

\begin{tabular}{|c|c|c|c|}
\hline Peak \# & R, Time & Area \% & Name \\
\hline 1. & 19.701 & 21.05 & n-Hexadecanoic acid \\
\hline 2. & 23.674 & 5.10 & Pentacosane \\
\hline 3. & 22.836 & 4.19 & Heneicosane \\
\hline 4. & 24.747 & 4.10 & Bis(2-ethylhexyl) phthalate \\
\hline 5. & 25.258 & 3.38 & Tetracontane \\
\hline 6. & 15.690 & 3.50 & Diethyl Phthalate \\
\hline 7. & 17.593 & 3.50 & Tetradecanoic acid \\
\hline 8. & 19.347 & 2.91 & Hexadecanoic acid, methyl ester \\
\hline 9. & 21.605 & 3.23 & Octadecanoic acid \\
\hline 10. & 36.316 & 4.51 & Beta-sitosterol \\
\hline 11. & 31.895 & 1.30 & Vitamin E \\
\hline 12. & 10.862 & 0.91 & 5-Hydroxymethylfurfural \\
\hline 13. & 11.193 & 0.32 & Bergamot mint oil \\
\hline 14. & 11.818 & 0.22 & Ascaridole epoxide \\
\hline 15. & 15.292 & 1.28 & Dodecanoic acid \\
\hline 16. & 16.031 & 0.39 & Carotol \\
\hline 17. & 27.243 & 0.45 & Squalene \\
\hline 18. & 18.449 & 1.14 & Neophytadiene \\
\hline 19. & 20.498 & 0.69 & Palmitic Acid, TMS derivative \\
\hline
\end{tabular}

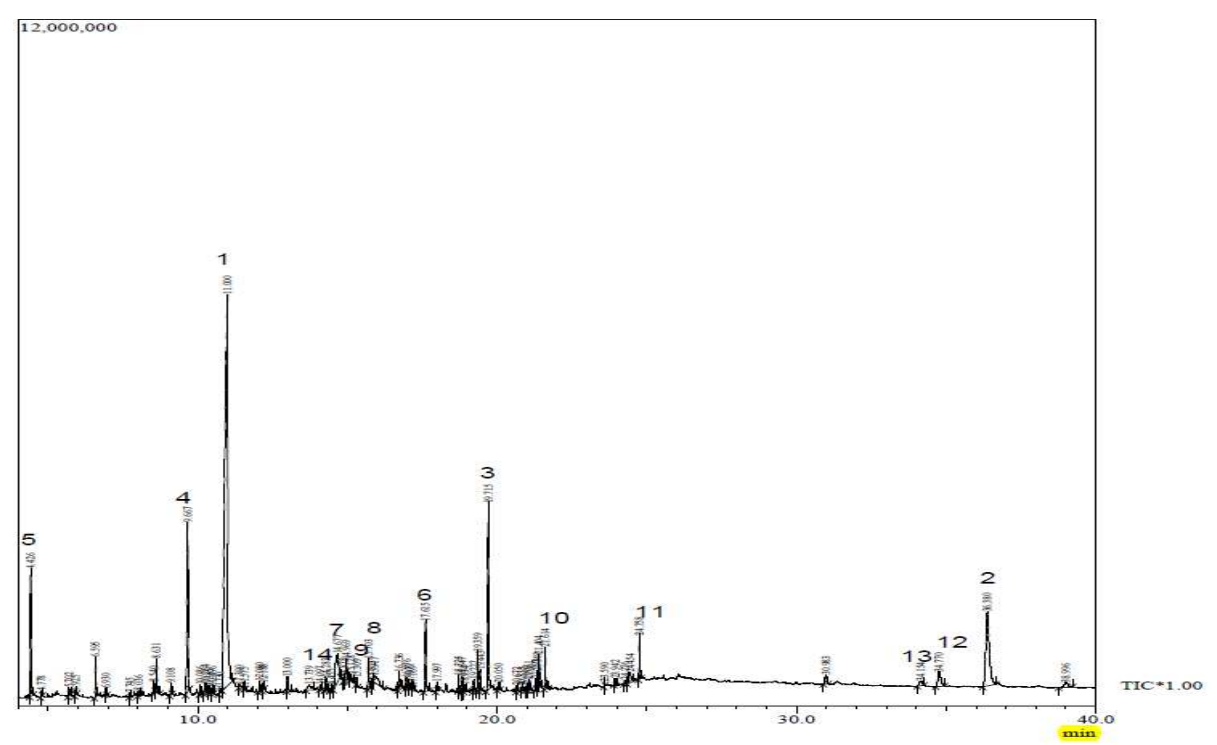

Fig. 4: GCMS chromatogram of methanolic callus extract of Ampelocissus latifolia

Table 2: Major peaks identified in in vitro methanolic extract of Ampelocissus latifolia

\begin{tabular}{|c|c|c|c|}
\hline Peak\# & R. Time & Area\% & Name \\
\hline 1. & 11.000 & 42.13 & 5-Hydroxymethylfurfural (HMF) \\
\hline 2. & 36.380 & 9.19 & Beta-Sitosterol \\
\hline 3. & 19.715 & 6.60 & n-Hexadecanoic acid \\
\hline 4. & 9.667 & 6.28 & 4H-Pyran-4-one, 2,3-dihydro-3,5-dihydroxy-6-methyl- \\
\hline 5. & 4.426 & 3.18 & Furfural \\
\hline 6. & 17.615 & 2.22 & Tetradecanoic acid \\
\hline 7. & 14.677 & 3.51 & D-Allose \\
\hline 8. & 15.703 & 0.94 & Diethyl Phthalate \\
\hline 9. & 14.969 & 1.06 & Caryophyllene \\
\hline 10. & 21.614 & 1.25 & Octadecanoic acid \\
\hline 11. & 24.758 & 1.11 & 1,2-Benzenedicarboxylic acid \\
\hline 12. & 34.770 & 1.64 & Stigmasterol \\
\hline 13. & 34.154 & 0.52 & Ergosterol \\
\hline 14. & 13.739 & 0.75 & Lactone $\mathrm{G}$ \\
\hline 15. & 15.309 & 0.19 & Dodecanoic acid \\
\hline 16. & 20.050 & 0.23 & Docosyl pentafluoropropionate \\
\hline 17. & 20.672 & 0.14 & Margarinic acid \\
\hline 18. & 21.297 & 0.24 & Methyl stearate \\
\hline 19. & 24.276 & 0.14 & 3-Hydroxypropyl palmitate, TMS derivative \\
\hline
\end{tabular}


Pharmaceutically and economically important phyto-components found in both in vivo and in vitro extracts and their biological activity

The present investigation was carried out to develop a protocol for in vitro callus induction of Ampelocissus latifolia (Roxb.) Planch. and to determine the bioactive compounds from its methanolic leaf and callus extract through GC-MS. The preliminary phytochemical studies of Ampelocissus latifolia showed the presence of carbohydrates, glycosides, tannins, alkaloids, saponins, flavonoids, steroids, phenols, proteins, hexose sugars, mucilages and gums [11]. Major compounds identified in both the extracts were Campesterol, Beta-sitosterol, Myristic acid, Stigmasterol, Diethyl phthalate, Propanoic acid, Malonic acid, Furyl hydroxymethyl alcohol, Butanedioic acid, Furfural, Linoleic acid, Caproic acid, Dodecanoic acid, Octadecanoic acid, etc. Few compounds were present only in callus extracts like Campesterol, Docosyl pentafluoropropionate, Lactone G, D allose, Dodecanol, Caryophyllene and Tocopherol, Squalene, Bergamol, Neophytadiene, only in leaf extracts. Phytoconstituents with maximum peak area in in vivo extract are Hexadecanoic acid (21.05\%), Pentacosane (5.10\%), $\beta$ sitosterol (4.51\%), Tocopherol (1.30\%) and 5-Hydroxymethylfurfural (42.13\%), $\beta$-sitosterol (9.19\%), Hexadecanoic acid (6.60\%), Stigmasterol (1.64\%). All identified compounds generally, were reported to have antimicrobial, antioxidant, anti-inflammatory and anti-cancerous activity and various applications in cosmetic and biofuel industry.

Stigmasterol is a phytosterol, used as a suitable precursor for semisynthetic progesterone manufacturing [12], as well as a precursor of vitamin $\mathrm{D}_{3}$ and related compounds [13]. Beta-Sitosterol is also a phytosterol, whose structure is similar to cholesterol and its consumption significantly lowers bad cholesterol levels. Pharmacological screening of $\beta$-sitosterol revealed various activities like antimicrobial, anti-inflammatory, anticancer, antifertility, angiogenic, antioxidant, immunomodulatory, antidiabetic, without major toxicity $[14,15]$. Campesterol is also a plant-derived sterol having structural similarity with cholesterol, thus competitively inhibits the absorption of cholesterol and may act in cancer prevention [16]. Docosanol is recently approved for pharmaceuticals by FDA (food and drug association) as an antiviral agent for reducing the pain and duration of cold sores caused by Herpes virus by tropical application [17]. Diethyl phthalate is used as Plasticizer. Linoleic acid is used in beauty products as an anti-inflammatory, anti-oxidant, acne reductive, skin lightening and moisture retentive agent on the skin [18]. Dodecanoic acid, Myristic acid, and Octadecanoic acid are used in soaps, and cosmetic industries and Lauric acid is used in the treatment of acne [19]. Hexadecanoic acid has been used in targeting the reduction of cardiovascular disease [20] obesity-related diseases and recently, cancer prevention [21]. Caryophyllene is a sesquiterpene which has anti-tumor, analgesic, antibacterial, anti-inflammatory, sedative, and fungicide activity [22]. Squalene has anti-cancerous properties and used as an adjuvant in vaccines and also in cosmetics [23]. Bergamot mint oil is used as essential oil in Aromatherapy and used for various health benefits [24] and Tocopherol also known as vitamin $\mathrm{E}$ has various beneficial effects for skin and human health [25]. Ascaridole epoxide was found to be having strong antimalarial and insecticidal activities [26]. 5-Hydroxymethylfurfural (HMF) has been found to bind specifically with intracellular sickle haemoglobin, for treatment of sickle cell disease [27] and is beneficial to human health by providing anti-oxidative, anti-allergic, anti-inflammatory, anti-hypoxic, and antihyperuricemic effects [28] Studies have showed that 5-HMF displayed higher antiproliferative activity on human melanoma A375 cells than other cell lines and could be developed as novel natural antioxidant with potential applications in cancer chemoprevention [29]. Neophytadiene is reported to have antifungal, antipyretic, antiinflammatory and analgesic properties [30].

\section{CONCLUSION}

It is the first report of in vitro callus induction in Ampelocissus latifolia and its comparative GC-MS analysis with that of in vivo leaf extract for identification of individual phytochemicals. From the results obtained through the in vitro callus induction and its comparative GC-MS analysis within vivo extract, it is revealed that Ampelocissus latifolia contains various bioactive compounds that are of importance for phytopharmaceutical uses. 63 different compounds were detected from leaf extract, and 59 phyto-compounds were observed from its callus extract. Few compounds were present only in callus extracts like Campesterol, Stigmasterol, Docosyl pentafluoropropionate, Lactone G, D allose, Dodecanol while Tocopherol, Squalene, Bergamol, were present only in leaf extracts. The GC-MS analysis revealed that the amount of Beta-sitosterol and 5-Hydroxymethylfurfural (HMF) was very high in in vitro extract as compared to in vivo extract. Majority of the common phytoconstituents like Hexadecanoic acid, Dodecanoic acid, Diethyl Pthalate, Octadecanoic acid, etc., were higher in leaf extract as compared to callus extract. Due to the identification of a large number of useful compounds in this species, the plant is considered phytopharmaceutical important for drug discovery and development.

\section{AUTHORS CONTRIBUTIONS}

All the authors have contributed equally

\section{CONFLICTS OF INTERESTS}

Declared none

\section{REFERENCES}

1. Maheswari J. Patenting Indian medicinal plants and products. Indian J Sci Technol 2011;4:298-301.

2. Nandagoapalan V, Doss A, Marimuthu C. Phytochemical analysis of some traditional medicinal plants. Bioscience Discovery 2016;7:17-20.

3. Jiang W, Myeong Je C, Lemaux PG. Improved callus quality and prolonged regenerability in the model and recalcitrant barley (Hordeum vulgare L.) cultivars. Plant Biotechnol 1998;15:63-9.

4. Anand DC, Patni V. Ampelocissus latifolia (Roxb.) Planch.-a traditional plant with enormous medicinal and economic importance. Int J Pharma Biosci 2016;7:303-7.

5. Tamilarasi CT, Subasini U, Kavimani S, Jaykar B. Phytochemical and pharmacological evaluation of Ampelocissus latifolia. Ancient Sci Life 2000;20:1-6.

6. Choudhury S, Choudhury HR, Mandal S. Pharmacognostic studies of Ampelocissus latifolia (Roxb.) Planch.-An Important Ethnomedicinal Plant. Bhubaneswar. Proceedings of the 99th session of the Indian Science Congress (II): Section XIV: Plant Sciences; 2012. p. 123.

7. Patil MV, Patil DA. Some herbal remedies used by the tribals of Nasik district, Maharashtra. Natl Prod Radiance 2007;6:152-7.

8. Chauhan A, Goyal MK, Chauhan P. GC-MS technique and its analytical applications in science and technology. J Anal Bioanal Tech 2014;5:222.

9. Ronald Hites A. Gas chromatography-mass spectroscopy: a handbook of instrumental techniques for analytical chemistry; 1997. p. 609-11.

10. Murashige T, Skoog F. A revised medium for rapid growth and bioassays with tobacco tissue cultures. Physiol Plant 1962; 15:473-97.

11. Pednekar PA, Kulkarni V, Raman B. Physicochemical and phytochemical evaluation of Ampelocissus latifolia (Roxb.) planch leaves. Int J Pharm Pharm Sci 2014;6:504-7.

12. Sundararaman P, Djerassi C. A convenient synthesis of progesterone from stigmasterol. J Org Chem 1977;42:3633-4.

13. Kametani T, Furuyama H. Synthesis of vitamin D3 and related compounds. Rev Article 1987;7:147-71.

14. Aringer L, Eneroth P, Nordstrom L. Side chain hydroxylation of cholesterol, campesterol and $\beta$-sitosterol in rat liver mitochondria. J Lipid Res 1976;17:263-72.

15. Awad AB, Chen YC, Fink CS, Hennessey T. $\beta$-sitosterol inhibits HT-29 human colon cancer cell growth and alters membrane lipids. Anticancer Res 1996;16:2797-804.

16. Genser B, Silbernagel G, De Backer G, Bruckert E, Carmena R, Chapman MJ, et al. Plant sterols and cardiovascular disease: a systematic review and meta-analysis. Eur Heart J 2012;33:444-51. 
17. Sacks SL, Thisted RA, Jones TM, Barbarash RA, Mikolich DJ, Ruoff GE, et al. Clinical efficacy of topical docosanol 10\% cream for herpes simplex labialis: a multicenter, randomized, placebocontrolled trial. J Am Acad Dermatol 2001;45:222-30.

18. Marounek M, Putthana V, Benada O, Lukesova D. Antimicrobial activities of medium-chain fatty acids and monoacylglycerols on Cronobacter sakazakii DBM 3157T and Cronobacter malonaticus DBM 3148. Czech J Food Sci 2012;30:573-80.

19. Yang D, Pornpattananangkul D, Nakatsuji T, Chan M, Carson D, Huang CM, et al. The antimicrobial activity of liposomal lauric acids against Propionibacterium acnes. Biomaterials 2009;30:6035-40.

20. Aranceta J, Perez Rodrigo C. Recommended dietary reference intakes, nutritional goals and dietary guidelines for fat and fatty acids: a systematic review. Br J Nutr 2012;107:S8-S22.

21. Berger NA. Obesity and cancer pathogenesis. Ann N Y Acad Sci 2014;1311:57-76.

22. Gopala Krishnan S. GC-MS analysis of some bioactive constituents of Mussaenda frondosa Linn. Int J Pharma Biosci 2011;2:313-20.

23. Huang Zih Rou, Lin Yin Ku, Fang Jia You. Biological and pharmacological activities of squalene and related compounds: potential uses in cosmetic dermatology. Molecules 2009;14: 540-54.

24. Romano L, Battaglia F, Masucci L, Sanguinetti M, Posteraro B, Plotti G, et al. In vitro activity of bergamot natural essence and furocoumarin-free and distilled extracts, and their associations with boric acid, against clinical yeast isolates. J Antimicrob Chemother 2005;55:110-4.

25. Rizvi S, Raza ST, Ahmed F, Ahmad A, Abbas S, Mahdi F. The role of vitamin e in human health and some diseases. Sultan Qaboos Univ Med J 2014;14:157-65.

26. Potawale SE, Luniya KP, Mantri RA, Mehta UK, Sadiq MW, Vetal YD. Chenopodium ambrosioides: an ethnopharmacological review. Pharmacol Online 2008;2:272-86.

27. Abdulmalik O, Safo MK, Chen Q Yang J, Brugnara C, OheneFrempong $\mathrm{K}$, et al. 5-hydroxymethyl-2-furfural modifies intracellular sickle haemoglobin and inhibits sickling of red blood cells. Br J Haematol 2005;128:552-61.

28. Zhao L, Chen J, Su J, Li L, Hu S, Li B, et al. In vitro antioxidant and antiproliferative activities of 5-hydroxymethylfurfural. J Agric Food Chem 2013;61:10604-11.

29. Li YX, Li Y, Qian ZJ, Kim MM, Kim SK. In vitro antioxidant activity of 5-HMF isolated from marine red alga Laurencia undulata in free-radical-mediated oxidative systems. J Microbiol Biotechnol 2009;19:1319-27.

30. Venkata Raman B, Samuel LA, Pardha Saradhi M, Narashimha Rao B, Naga Vamsi Krishna A, Sudhakar M, et al. Antibacterial, antoxidant activity and GC-MS analysis of Eupatorium odoratum. Asian J Pharm Clin Res 2012;5:99-106. 